\title{
Phase readout of a charge qubit capacitively coupled to an open double quantum dot
}

\author{
Christoph Kreisbeck, ${ }^{1,2}$ Franz J. Kaiser, ${ }^{2}$ and Sigmund Kohler ${ }^{3}$ \\ ${ }^{1}$ Institut für Theoretische Physik, Universität Regensburg, D-93040 Regensburg, Germany \\ ${ }^{2}$ Institut für Physik, Universität Augsburg, Universitätsstraße 1, D-86135 Augsburg, Germany \\ ${ }^{3}$ Instituto de Ciencia de Materiales de Madrid, CSIC, Cantoblanco, E-28049 Madrid, Spain
}

(Dated: December 4, 2018)

\begin{abstract}
We study the dynamics of a charge qubit that is capacitively coupled to an open double quantum dot. Depending on the qubit state, the transport through the open quantum dot may be resonant or off-resonant, such that the qubit affects the current through the open double dot. We relate the initial qubit state to the magnitude of an emerging transient current peak. The relation between these quantities enables the readout of not only the charge but also the phase of the qubit with sufficient resolution.
\end{abstract}

PACS numbers: 42.50.Dv, 73.23.-b, 03.67.Lx, 05.60.Gg

\section{INTRODUCTION}

Quantum algorithms usually terminate with qubit readout, i.e., a measurement of the quantum register's state. Generally, the laws of quantum mechanics inhibit one to directly and fully determine the wave function of the qubit from a single measurement. With repeated projective measurements in the same basis, it is only possible to sample the probability that the qubit is in the one or the other of two orthogonal states. Such destructive measurements can nevertheless be used to demonstrate coherent oscillations by repeating the experiment many times. Such experiments have been performed for superconducting qubits,,$\underline{\underline{\underline{3}}}$ as well as for charge qubits implemented with double quantum dots $\underline{\underline{4}} \underline{\underline{6}}$ Recently, the coupling of two charge qubits of the latter type has been demonstrated,,$\underline{7}$ which represents a first step toward performing gate operations.

In order to distinguish in an experiment between different charge states of a single quantum dot, one may couple the dot capacitively to a quantum point contact which acts as charge meter. Then the current through the meter depends on the number of electrons in the quantum dot. This also allows one to monitor the transport of individual electrons ${ }^{8}$ and to eventually determine the associated full counting statistics $\underline{9}$ It has also been proposed to couple a charge qubit to a point contact, such that the current through the latter depends on the location of the electron in the double quantum dot, i.e., on the state of a charge qubit $\stackrel{10,11}{=}$ It is also possible to employ a voltage-biased open quantum dot as charge meter if a nearby additional charge shifts one energy level of the quantum dot across the Fermi surface of an attached lead, the current depends as well on the presence of the charge 12 When measuring the state of a charge qubit in that way, the measurement acts back on the coherence of the qubit which, thus, experiences decoherence and dissipation. This means that the qubit evolves into an incoherent mixture. The associated transient current allows one to infer on the initial charge state of the qubit.

A double quantum dot can be used as charge meter as well $\stackrel{13}{\underline{15}}$ There the main idea is that the monitored

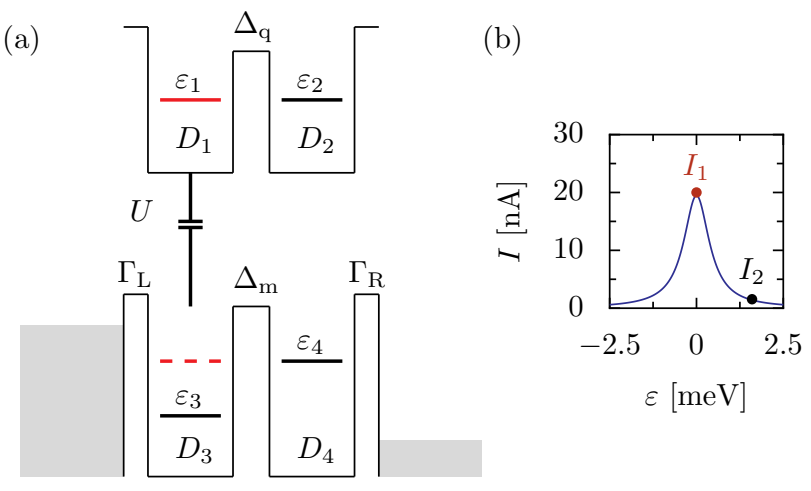

FIG. 1: (Color online) (a) Sketch of the qubit-meter setup consisting of an open and a closed double quantum dot. The closed double dot $\left(D_{1}\right.$ and $\left.D_{2}\right)$ is occupied with one electron and forms a charge qubit. An electron in dot $D_{1}$ effectively shifts the onsite energy of $\operatorname{dot} D_{3}$, such that dots $D_{3}$ and $D_{4}$ are tuned into resonance. Thus the current through the open double dot $\left(D_{3}\right.$ and $\left.D_{4}\right)$ is sensitive to the state of the qubit. (b) Current as a function of the meter energy bias $\varepsilon=\varepsilon_{4}-\varepsilon_{3}$ without coupling to the qubit, $U=0$, for inter-dot tunneling $\Delta_{\mathrm{m}}=0.2 \mathrm{meV}$ and wire-lead coupling $\Gamma=0.2 \mathrm{meV}$. The marked values correspond to the energy shift induced by a qubit electron localized in $\operatorname{dot} D_{1}$ and $D_{2}$, respectively, for the interaction strength $U=1 \mathrm{meV}$.

charge acts as gate voltage on one quantum dot, such that the energy levels of the double dot are tuned into resonance. The consequence is that the conductance of the double dot and, thus, the current increases, see Fig. 1(b). For the parameters of this figure, the current changes by roughly a factor 10 upon qubit tunneling from $\operatorname{dot} D_{2}$ to $\operatorname{dot} D_{1}$. Thus the achievable signal-to-noise ratio for this charge measurement is higher than the one for the singledot meter $\stackrel{14,15}{1}$ Moreover, different qubit states lead to significantly different currents, such that the measurement basis is not fluctuating. ${ }^{11,16}$ The central aim of this work is to demonstrate that the double-dot charge meter can even be used to distinguish between qubit states with identical population but different phase. This means that 
one can read out either the charge or the phase of the qubit, depending on the choice of the experimenter. The phase readout proposed below corresponds to the measurement of an observable that does not commute with the system-meter coupling Hamiltonian, ${ }^{17,18}$

\section{QUBIT COUPLED TO METER}

The setup sketched in Fig. 1is described by the Hamiltonian

$$
H=H_{\mathrm{q}}+H_{\mathrm{m}}+H_{\mathrm{q}-\mathrm{m}}+H_{\mathrm{m}-\mathrm{l}}+H_{\text {leads }},
$$

where the first two terms describe the closed and the open double quantum dot forming the qubit and the meter, respectively. They read

$$
\begin{aligned}
H_{\mathrm{q}} & =\Delta_{\mathrm{q}}\left(c_{1}^{\dagger} c_{2}+c_{2}^{\dagger} c_{1}\right)=\Delta_{\mathrm{q}} \sigma_{x}, \\
H_{\mathrm{m}} & =\varepsilon_{3} n_{3}+\varepsilon_{4} n_{4}+\Delta_{\mathrm{m}}\left(c_{3}^{\dagger} c_{4}+c_{4}^{\dagger} c_{3}\right),
\end{aligned}
$$

i.e., each dot $D_{i}$ is described by a single level with onsite energy $\varepsilon_{i}$ and the usual fermionic creation and annihilation operators of an electron, $c_{i}^{\dagger}$ and $c_{i}$, with the corresponding electron number $n_{i}=c_{i}^{\dagger} c_{i}$. For the present purpose, it is sufficient to consider spinless electrons, such that each dot can be occupied by at most one electron. $\Delta_{\mathrm{q}}$ and $\Delta_{\mathrm{m}}$ are the inter-dot tunnel matrix elements of the closed and the open double dot, respectively. Since the closed double dot is occupied with a single electron, we can write the Hamiltonian (2) in pseudo-spin notation with the basis states $\left|D_{1,2}\right\rangle=c_{1,2}^{\dagger}|0\rangle_{\mathrm{q}}$ and the usual Pauli matrices $\sigma_{x, y, z}$. We restrict ourselves to a qubit Hamiltonian without bias term $\propto \sigma_{z}$ since such term can always be removed by proper gate voltages.

Electrons residing in dots $D_{1}$ and $D_{3}$ interact capacitively with strength $U$ according to

$$
H_{\mathrm{q}-\mathrm{m}}=U n_{1} n_{3} .
$$

Notice that this qubit-meter coupling does not commute with the qubit Hamiltonian (21). Thus the measurement is destructive and, in particular, not of quantum non-demolition type, such that the analysis proposed in Ref. 19 is not applicable here.

The meter is in addition coupled to two metallic leads which we model as free electron gases with the Hamiltonian

$$
H_{\text {leads }}=\sum_{\ell=\mathrm{L}, \mathrm{R}} \sum_{k} \hbar \omega_{\ell k} c_{\ell k}^{\dagger} c_{\ell k}
$$

where the operator $c_{\ell k}^{\dagger}$ creates an electron in state $k$ of lead $\ell=\mathrm{L}, \mathrm{R}$ with energy $\hbar \omega_{\ell k}$. Henceforth, we consider the limit of large bias voltage such that initially all relevant states of the left lead are occupied, while those of the right lead are empty. Then the electron transport becomes unidirectional. Electron tunnelling between the leads and the open double dot is described by the Hamiltonian

$$
H_{\mathrm{m}-\mathrm{l}}=\sum_{k}\left(V_{\mathrm{L} k} c_{\mathrm{L} k}^{\dagger} c_{3}+V_{\mathrm{R} k} c_{\mathrm{R} k}^{\dagger} c_{4}\right)+\text { H.c. }
$$

The coupling matrix elements $V_{\ell k}$ can be subsumed in the effective tunnel rates $\Gamma_{\ell}=(2 \pi / \hbar) \sum_{\ell, k}\left|V_{\ell k}\right|^{2} \delta\left(\epsilon-\epsilon_{\ell k}\right)$, which within a wide-band limit are assumed to be energy independent.

In order to compute the time evolution of the systemmeter setup, we derive a master equation for the reduced density operator $\rho$ of both double quantum dots by eliminating the leads within second-order perturbation theory. Starting from the Liouville-von Neumann equation $\dot{R}(t)=-(i / \hbar)[H, R(t)]$ for the total density operator $R$, we follow Ref. 20 and obtain by tracing out the leads the master equation,

$$
\begin{aligned}
\dot{\rho}(t)= & -\frac{\mathrm{i}}{\hbar}\left[H_{\mathrm{s}}, \rho(t)\right]-\frac{1}{\hbar^{2}} \int_{0}^{\infty} d \tau \operatorname{Tr}_{\text {leads }} \\
& \times\left\{\left[H_{\mathrm{m}-\mathrm{l}},\left[\tilde{H}_{\mathrm{m}-\mathrm{l}}(-\tau), \rho(t) \otimes \rho_{\text {leads }}\right]\right]\right\},
\end{aligned}
$$

with $\rho_{\text {leads }}$ being the density operator of both leads, each in a canonical state but with different Fermi energy. The factorization assumption for the density operator allows evaluating the trace over the lead states such that a closed equation for the reduced density operator of the quantum dots remains. The first term on the right-hand side refers to the coherent dynamics of the electrons in the two coupled double dots, while the second term describes incoherent tunnelling between the leads and the open double quantum dot. The tilde denotes the interaction picture with respect to the system Hamiltonian $H_{\mathrm{s}}=H_{\mathrm{q}}+H_{\mathrm{m}}+H_{\mathrm{q}-\mathrm{m}}$. Defining the current operator in a symmetric manner, $\mathcal{J}=(e / 2)\left(\dot{N}_{\mathrm{L}}-\dot{N}_{\mathrm{R}}\right)$, we obtain the ensemble-averaged expectation value $I(t)=$ $(e / 2) \operatorname{Tr}\left\{\left(\mathcal{J}_{\mathrm{L}}^{\text {in }}+\mathcal{J}_{\mathrm{R}}^{\text {out }}\right) \rho(t)\right\}$. In the large-bias limit, the superoperators $\mathcal{J}_{\mathrm{L}}^{\text {in }}, \mathcal{J}_{\mathrm{R}}^{\text {out }}$ act on the reduced density operator according to

$$
\begin{aligned}
\mathcal{J}_{\mathrm{L}}^{\text {in }} \rho & =\Gamma_{\mathrm{L}} c_{3}^{\dagger} \rho c_{3}, \\
\mathcal{J}_{\mathrm{R}}^{\text {out }} \rho & =\Gamma_{\mathrm{R}} c_{4} \rho c_{4}^{\dagger} .
\end{aligned}
$$

They describe incoherent tunnelling of an electron from the left lead to dot $D_{3}$ and from $\operatorname{dot} D_{4}$ to the right lead, respectively. Notice that for large bias voltage, the meter properties no longer depend on the absolute values of the onsite energies $\varepsilon_{3}$ and $\varepsilon_{4}$, but only on the energy bias $\varepsilon=\varepsilon_{4}-\varepsilon_{3}$. All numerical results presented below have been obtained by integrating master equation (7).

\section{MEASUREMENT CONCEPT AND VISIBILITY}

The central idea of our readout scheme is that the current through the open double dot depends on the position of the electron in the closed quantum dot, i.e., on 
the state of the qubit. The qubit in turn is affected by the coupling to the leads via the meter which thus effectively represents a macroscopic environment. Therefore, the qubit will experience decoherence, such that the qubit-meter setup will evolve into a generally unique stationary mixed state in which the current assumes a value independent of the initial condition. This transition corresponds to a "collapse" of the wave function during a finite time. Moreover, it implies that the readout is destructive, i.e., some information about the qubit state is transfered to the leads. The full quantum state of the lead, however, is not accessible such that the measurement is quantum limited. Nevertheless, measurement of macroscopic lead observables such as the current is possible. In the present case, the current exhibits transients that allow one to draw conclusions on the qubit's initial state which we propose to read out. In the following, we reveal the underlying relation between the initial qubit state and the transient current.

As a natural and experimentally relevant initial condition, we assume that in the beginning, the meter is not coupled to the qubit $(U=0)$ and stays in the corresponding stationary state which is a mixed non-equilibrium state in which a current flows ${ }^{21}$ At this stage, the qubit is in a pure state which we parameterize on the Bloch sphere as

$$
|\psi(t=0)\rangle_{\mathrm{q}}=\cos (\theta / 2)\left|D_{1}\right\rangle+e^{i \phi} \sin (\theta / 2)\left|D_{2}\right\rangle,
$$

where the state $\left|D_{1,2}\right\rangle$ refers to an accordingly localized electron. The angle $\theta=0 \ldots \pi$ determines the position $\left\langle\sigma_{z}\right\rangle=\left\langle n_{1}\right\rangle-\left\langle n_{2}\right\rangle=\cos \theta$ of the electron, while $\phi=0 \ldots 2 \pi$ denotes the relative phase. State preparation with such qubits has been demonstrated experimentally in Refs. 4, 5. At time $t=0$, the qubit-meter coupling $U$ is switched on, such that the qubit influences the current and the readout process starts.

Before addressing phase readout, we elucidate the underlying mechanism for the more intuitive charge readout $10,14,15$ Figures 2 (a,b) show a typical time evolution of the qubit population and the corresponding current. If the qubit is initially in state $\left|D_{2}\right\rangle$, i.e. for $\theta=\pi$, it will essentially stay there and, thus, the open double dot remains off-resonant and the current small. This stems from the fact that for a given $U$, the energy of the two double dots is smaller when the qubit electron resides in one particular dot, which for the present parameters is $\operatorname{dot} D_{2}$. This implies that the initial state $\left|D_{2}\right\rangle$ is already close to the stationary state and not much dynamics is going on. When starting in state $\left|D_{1}\right\rangle(\theta=0)$, by contrast, the capacitive coupling tunes the levels of dots $D_{3}$ and $D_{4}$ into resonance and the current starts to increase until the systems evolves into a stationary state with an again smaller current. Thus, we observe a current peak. The solid line in Fig. 2(c) shows that the peak current $\hat{I}$, i.e. the maximal current, is related to the population parameter $\theta$. This clear dependence is quite important for the readout scheme, because it implies that the measurement of $\hat{I}$ corresponds to the determination of the
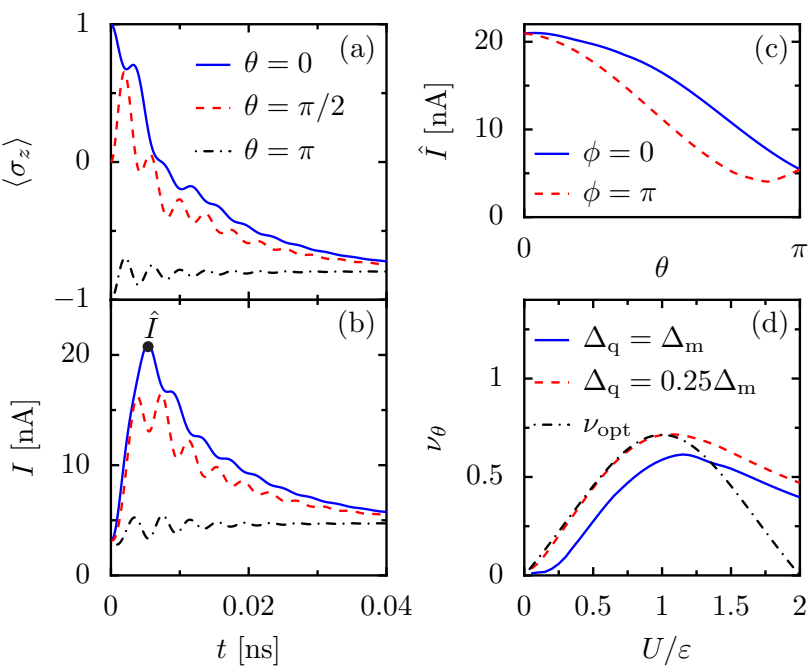

FIG. 2: (Color online) Charge readout for $\Gamma_{\mathrm{L}}=\Gamma_{\mathrm{R}}=$ $0.2 \mathrm{meV}, \Delta_{\mathrm{q}}=\Delta_{\mathrm{m}}=0.2 \mathrm{meV}$, and $U=\varepsilon=1 \mathrm{meV}$. (a) Transient dynamics of the qubit population $\left\langle\sigma_{z}\right\rangle=\left\langle n_{1}-n_{2}\right\rangle$ and (b) corresponding current for various initial localizations. (c) Height of the current peak as a function of the initial occupation $\cos (\theta / 2)$ for different relative phases $\phi$. (d) Chargereadout visibility $\nu_{\theta}$. The dashed-dotted line marks the "optimal" visibility, for which backaction is ignored; see Fig. 1(b).

expectation value $\left\langle\sigma_{z}\right\rangle=\cos \theta$ for the initial qubit state.

Readout with good resolution requires that the peak height depends strongly on the initial population. A figure of merit for this is the scaled difference between the maximum and the minimum of $\hat{I}$ upon variation in $\theta$, i.e., the charge-readout visibility defined as

$$
\nu_{\theta}=\frac{\max _{\theta} \hat{I}-\min _{\theta} \hat{I}}{\max _{\theta} \hat{I}+\min _{\theta} \hat{I}} .
$$

Figure 2(d) shows this quantity for two different qubit splittings. The visibility is best $\left(\nu_{\theta} \approx 0.75\right)$ when the interaction energy $U$ matches the bias $\varepsilon$ of the meter and the qubit dynamics is slower than electron tunnelling through the meter, i.e., when $\Delta_{\mathrm{q}}<\Delta_{\mathrm{m}}, \Gamma_{\mathrm{L}, \mathrm{R}}$. For $U \lesssim \varepsilon$, the visibility even reaches the optimal value which is achieved when backaction of the meter to the qubit is ignored. This complies with the picture drawn by investigating the peak-to-background ratio. 14,15

Most interestingly, Fig. 2(c) indicates that the peak current $\hat{I}$ for a given initial population also depends on the initial relative phase $\phi$. This already hints on the feasibility of phase readout, despite the fact that the difference in $\hat{I}$ is not very pronounced for the parameters used here.

\section{PHASE-READOUT}

We have already seen in the last section that phase readout is possible in principle. This raises two intrigu- 
ing questions. First, we would like to qualitatively understand why phase readout works, despite the fact that the capacitive coupling is sensitive to the location of the qubit electron only. The second question is of quantitative nature: can one achieve a visibility comparable to the one obtained for charge readout?

The relative phase is only meaningful when both qubit states are populated, and a noticeable influence on the transient current requires even significant population of both states. Therefore, we restrict ourselves to equal initial population, i.e., to $\theta=\pi / 2$. In order to gain a physical picture of the phase readout, let us focus on the two phases $\phi=\pi$ and $\phi=0$, i.e., on the states $\left(\left|D_{1}\right\rangle-\left|D_{2}\right\rangle\right) / \sqrt{2}$ and $\left(\left|D_{1}\right\rangle+\left|D_{2}\right\rangle\right) / \sqrt{2}$, which are the eigenstates of the qubit Hamiltonian (2) in the oneelectron subspace. Since the qubit couples via the meter to a macroscopic environment, it will evolve into an asymptotic state. Thus, for the ground state $(\phi=\pi)$, the qubit will absorb energy from the meter, while for the excited state $(\phi=0)$, the qubit emits energy. Both processes leave their fingerprints in the transients of the current and, thus, allow one to discern different initial phases.

A typical transient current is shown together with the corresponding qubit dynamics in Figs. 3(a,b). The current peak resembles the one analyzed in the context of charge readout. Here however, the peak never vanishes completely, as it was the case above for the initial state $\left|D_{2}\right\rangle$. Thus, at first glance, phase readout seems to possess a much lower resolution than charge readout. For a quantitative analysis we consider the phase-readout visibility $\nu_{\phi}$ which we define according to Eq. (11) but with the population parameter $\theta$ replaced by the relative phase $\phi$.

Figure 3)(c) reveals the clear $\phi$-dependence of the current peak height $\hat{I}$, which forms the basis of our phasereadout scheme. The maximal value and the minimal value of $\hat{I}$ are assumed for phases very close to $\phi=0$ and $\phi=\pi$, respectively. Therefore the experimental distinction between these two phases can be achieved with the full visibility $\nu_{\phi}$ shown in Fig. 3(d). As compared to charge readout, the visibility exhibits a less regular structure. For the interaction strength $U \approx \varepsilon / 2$, it reaches a value $\nu_{\phi} \approx 0.25$ and remains of that order when $U$ is increased. In contrast to charge readout, we find a tendency toward higher visibility for larger qubit splitting. Nevertheless, for these parameters, $\nu_{\phi}$ still stays clearly below the charge readout visibility $\nu_{\theta}$. Therefore it is essential to optimize the setup.

Three routes toward an optimized phase readout come to mind. First, as already noticed above, the qubit splitting $\Delta_{\mathrm{q}}$ should be larger than the tunnel matrix element $\Delta_{\mathrm{m}}$ of the meter. Irrespective of any experimental constraints, increasing $\Delta_{\mathrm{q}}$ is only of limited use, because beyond a certain limit, the qubit oscillations then become so fast that the meter is no longer able to follow. Consequently, the meter no longer contains information on the qubit and, thus, the readout quality will decrease. In
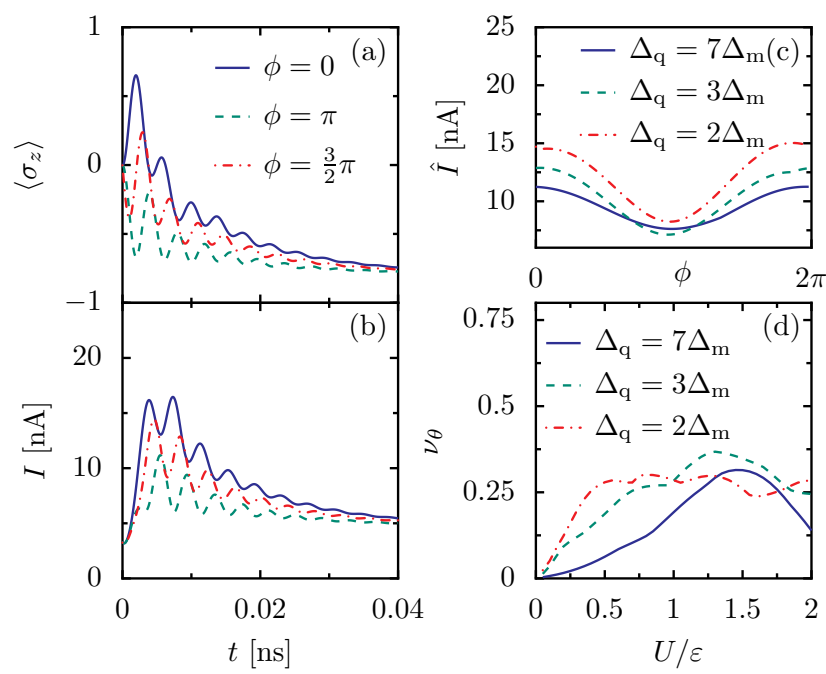

FIG. 3: (Color online) Phase readout of a qubit with energy splitting $\Delta_{\mathrm{q}}=0.2 \mathrm{meV}$ coupled to a meter with the same parameters as in Fig. 2. The qubit states are at initial time equally populated, i.e., $\theta=\pi / 2$. (a) Transient qubit dynamics and (b) current for various initial phases $\phi$ and qubit-meter interaction $U=\varepsilon=1 \mathrm{meV}$ and $\Delta_{\mathrm{q}}=0.2 \mathrm{meV}$. (c) Height of the current peak as a function of the initial phase. (d) Readout visibility as a function of the qubit-meter interaction strength $U$.

our case, we find that $\Delta_{\mathrm{q}}=3 \Delta_{\mathrm{m}}$ is a good choice, while for larger qubit splittings, the visibility indeed decreases; see Fig. 3(d).

A second way for improving the visibility is to tune the meter into a regime of higher sensitivity which is mainly determined by the resonance curve shown in Fig. 1(b). When the dot-lead tunnel rates $\Gamma_{\mathrm{L}, \mathrm{R}}$ become smaller, the current maximum $I_{1}$ increases, while the current for an energy bias $\varepsilon=U$, which is $I_{2}$, decreases. Consequently, the achievable visibility $\nu_{\text {opt }}$ becomes larger. The reduced dot-lead tunnelling, however, leads to a smaller current, such that the current measurement eventually will be difficult.

Alternatively, one can reduce the current $I_{2}$ by using a setup with a larger bias $\varepsilon$ and an accordingly larger interaction energy $U$. The Coulomb interaction $U$, however, is determined by the distance between the dots $D_{1}$ and $D_{3}$ and, thus, is limited by the size of the top gates that define the quantum dots. Nevertheless, it is possible to enhance the qubit-meter coupling by choosing a setup in which also the electrons in dots $D_{2}$ and $D_{4}$ repel each other. Then the qubit-meter Hamiltonian (4) has to be extended by the term $U^{\prime} n_{2} n_{4}$. If now an electron tunnels from $D_{2}$ to $D_{1}$, the left meter level is raised by $U$, while the right meter level is no longer raised by $U^{\prime}$, i.e., it is effectively lowered by $U^{\prime}$. This implies that the relevant effective interaction strength is $U_{\text {eff }}=U+U^{\prime}$. Notice that the resonance condition for the meter bias nevertheless reads $\varepsilon=U$. This additional qubit-meter coupling represents our third way of optimization. We explore its ben- 

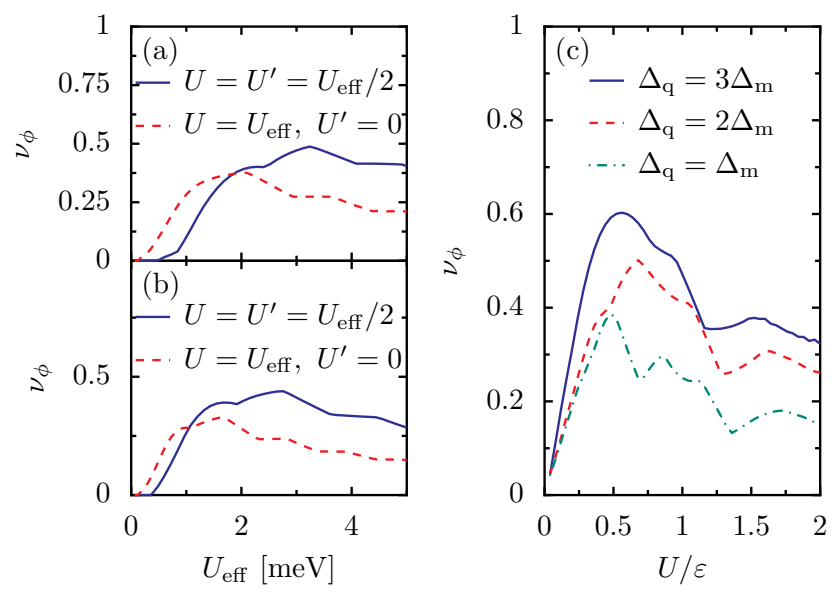

FIG. 4: (Color online) Phase-readout visibility for a meter with $\Gamma_{\mathrm{L}}=\Gamma_{\mathrm{R}}=0.2 \mathrm{meV}$ and tunnel matrix element $\Delta_{\mathrm{m}}=0.2 \mathrm{meV}$. Comparison of symmetric coupling $(U=\varepsilon)$ and coupling between the left dots $\left(U^{\prime}=0\right)$ for qubit energy splitting (a) $\Delta_{\mathrm{q}}=3 \Delta_{\mathrm{m}}$ and (b) $\Delta_{\mathrm{q}}=2 \Delta_{\mathrm{m}}$. (c) Visibility as a function of the interaction strength $U$ for symmetric coupling $U^{\prime}=U$, bias $\varepsilon=1.5 \mathrm{meV}$ and various qubit splittings.

efits by comparing two situations with the same effective coupling strength: symmetric coupling $U=U^{\prime}=U_{\text {eff }} / 2$ and coupling only between $\operatorname{dots} D_{1}$ and $D_{3}$, which means $U=U_{\text {eff }}$ while $U^{\prime}=0$.

Figures $4(a, b)$ show the resulting visibilities as a function of the meter bias. We find that in the relevant regime with large $\nu_{\phi}$, the symmetric coupling is superior to the asymmetric coupling. The difference is up to roughly $30 \%$. Finally, in order to explore the limits of the symmetrically coupled setup, we plot in Fig. 4(c) the visibility as a function of the interaction strength. It turns out that the result is best in the vicinity of $U=\varepsilon / 2$. The observed maximum is rather broad, which means that our readout scheme is quite tolerant against moderate parameter variations. Summarizing our optimization procedure, we find that the phase-readout visibility can be up to $\nu_{\phi}=0.6$, which is smaller but still of the same order as what we found for charge readout $\left(\nu_{\theta}=0.75\right)$. This clearly demonstrates the feasibility of phase readout.

For the numbers used in our study, the current peaks are on the order of $10 \mathrm{nA}$ with a duration of up to $10 \mathrm{~ns}$. Thus a peak typically consists of ten electrons. Assuming that the transported electrons are uncorrelated, they obey Poisson statistics and, thus, the standard deviation of the number of transported electrons is \pm 3 . This deviation should represent the relevant fluctuation of the signal. Owing to these numbers, the experimental realization of our phase readout (and of related chargereadout schemes $12,14,15$ as well) represents a demanding task. Nevertheless, it should be feasible, e.g., by repeating preparation and readout many times, such that the current peaks turn into an enhanced average current. ${ }^{4.5}$
Alternatively, one could think of combining the charge meter with setups for monitoring the transport of individual electrons .8 .9

At the readout stage of a quantum algorithm, one already knows that the qubit resides in either of two specific orthogonal states $\stackrel{22}{=}$ If these states are the charge states $\left|D_{1}\right\rangle$ and $\left|D_{2}\right\rangle$, the results of Sec. III allow one to distinguish between them. The results of the present section, by contrast, are of use when the two possible final states of a gate operation are given by Eq. (10) for $\theta=\pi / 2$ and for example $\phi=0, \pi$.

\section{CONCLUSIONS}

We have investigated the transient current through an open double quantum dot with a capacitive coupling to a charge qubit. In particular, we focused on the impact of the initial qubit state on the current peak that emerges after the qubit is coupled to the open double quantum dot. Such qubit-meter setups have recently been proposed for monitoring the location of an electron in a closed double dot, i.e., for charge readout with single or double dot meters. Our results demonstrate that such a charge meter is useful for phase readout as well, despite the fact that the qubit phase possesses only indirect influence on the measured current. For an unbiased qubit, the relative phase determines whether the qubit is initially in its ground state or in its excited state. Thus, when coupled to a macroscopic device such as the meter, the qubit will absorb or emit energy, depending on its initial phase. This difference is visible in the height of a transient current peak, whose measurement thus corresponds to phase readout.

After having realized that phase readout is possible, in principle, we have investigated whether the measured signals are sufficiently pronounced, such that they allow one to reliably discern between different initial phases. Our results for the phase readout visibility, defined as the scaled difference of the current peaks, reveal that phase readout is only slightly more demanding than the previously proposed charge readout with single or double quantum dot meters. In conclusion, we believe that the experimental implementation of our phase-readout scheme opens a promising way for the observation of coherent tunnelling dynamics in double quantum dots.

\section{Acknowledgments}

This work has been supported by the DFG through SFB 631 and by the German Excellence Initiative via "Nanosystems Initiative Munich (NIM)". S.K. acknowledges support by the Spanish Ministerio de Ciencia e Innovación through the Ramón y Cajal program. 
1 Y. Nakamura, Y. A. Pashkin, and J. S. Tsai, Nature (London) 398, 786 (1999).

2 D. Vion, A. Aassime, A. Cottet, P. Joyez, H. Pothier, C. Urbina, D. Esteve, and M. H. Devoret, Science 296, 886 (2002).

3 I. Chiorescu, Y. Nakamura, C. J. P. Harmans, and J. E. Mooij, Science 299, 1869 (2003).

4 T. Hayashi, T. Fujisawa, H. D. Cheong, Y. H. Jeong, and Y. Hirayama, Phys. Rev. Lett. 91, 226804 (2003).

5 T. Fujisawa, T. Hayashi, H. D. Cheong, Y. H. Jeong, and Y. Hirayama, Physica E 21, 1046 (2004).

6 J. Gorman, D. G. Hasko, and D. A. Williams, Phys. Rev. Lett. 95, 090502 (2005).

7 K. D. Petersson, C. G. Smith, D. Anderson, P. Atkinson, G. A. C. Jones, and D. A. Ritchie, Phys. Rev. Lett. 103, 016805 (2009).

8 S. Gustavsson, R. Leturcq, B. Simovič, R. Schleser, T. Ihn, P. Studerus, K. Ensslin, D. C. Driscoll, and A. C. Gossard, Phys. Rev. Lett. 96, 076605 (2006).

9 C. Fricke, F. Hohls, W. Wegscheider, and R. J. Haug, Phys. Rev. B 76, 155307 (2007).

10 H.-S. Goan, G. J. Milburn, H. M. Wiseman, and H. B. Sun, Phys. Rev. B 63, 125326 (2001).

11 S. Ashhab, J. Q. You, and F. Nori, New J. Phys. 11, 083017 (2009).

12 H. M. Wiseman, D. Wahyu Utami, H. B. Sun, G. J. Milburn, B. E. Kane, A. Dzurak, and R. G. Clark, Phys. Rev.
B 63, 235308 (2001).

13 T. Geszti and J. Z. Bernád, Phys. Rev. B 73, 235343 (2006).

14 T. Gilad and S. A. Gurvitz, Phys. Rev. Lett. 97, 116806 (2006).

15 H. J Jiao, X.-Q. Li, and J. Y. Luo, Phys. Rev. B 75, 155333 (2007)

16 S. Ashhab, J. Q. You, and F. Nori, Phys. Scr. T137, 014005 (2009).

17 G. M. Reuther, D. Zueco, P. Hänggi, and S. Kohler, Phys. Rev. Lett. 102, 033602 (2009).

18 S. Ashhab, J. Q. You, and F. Nori, Phys. Rev. A 79, 032317 (2009).

19 J. Gambetta, W. A. Braff, A. Wallraff, S. M. Girvin, and R. J. Schoelkopf, Phys. Rev. A 76, 012325 (2007).

${ }^{20}$ F. J. Kaiser and S. Kohler, Ann. Phys. (Leipzig) 16, 702 (2007).

21 We also performed calculations with other initial conditions, such as an empty meter double quantum dot. Irrespective of their experimental feasibility, it is interesting to note that the results are similar to those presented below and lead to the same conclusions.

22 M. A. Nielsen and I. L. Chuang, Quantum Computing and Quantum Information (Cambridge University Press, Cambridge, 2000). 